\title{
PHENOMENOLOGICAL VARIANT OF QUASI-CHEMICAL APPROXIMATION IN THE DESCRIPTION OF THE DIMERISATION OF ADSORBATE MOLECULES IN A MULTILAYER ADSORPTION PHASE ON A HOMOGENEOUS SURFACE
}

\author{
Jerzy K. Garbacz ${ }^{1)}$, Mariusz Kozakiewicz ${ }^{2)}$, Barbara Rymian ${ }^{3)}$ \\ 1) University of Technology and Life Sciences in Bydgoszcz, Department of Environment Engineering, Poland \\ 2) Nicolaus Copernicus University in Torun L. Rydygier Collegium Medicum in Bydgoszcz, Food Chemistry Department, Poland \\ 3) Nicolaus Copernicus University in Torun L. Rydygier Collegium Medicum in Bydgoszcz Department of Hygiene, Epidemiology and Ergonomics
}

\section{ABSTRACT}

This article is related thematically to two of our earlier publications, which demonstrated full equivalence of statistical and phenomenological methods in the description of physical gas adsorption on the surface of a solid body, and the fundamental possibility of analytical solution of adsorbate-adsorbate association problems in the entire multi-layer adsorption phase. The quasi-chemical scheme of secondary interactions leading to the formation of horizontal multimolecular adsorption complexes has been elaborated. A new adsorption equation was formulated taking into account the dimerisation of adsorbed molecules in the whole adsorption phase, as well as the influence of topography of the binding sites of adsorbent surfaces on the form of this solution. Keywords: adsorption, surface homogeneity, adsorbate-adsorbate association, adsorption multiple layers.

\section{ARTICLE INFO}

PolHypRes 2019 Vol. 68 Issue 3 pp. $33-40$

ISSN: 1734-7009 elSSN: 2084-0535

DOI: $10.2478 /$ phr-2019-0011

Pages: 8 , figures: 1 , tables: 1

page www of the periodical: www.phr.net.pl

Publisher

Polish Hyperbaric Medicine and Technology Society

\section{Original article}

Submission date: $19.05 .2019 \mathrm{r}$

Acceptance for print: 19.07.2019 r. 


\section{INSTRODUCTION}

The proposal for the description of adsorbateadsorbate association presented in our previous publications [1,2] resulted in new equations for the adsorption of a single gas on a homogeneous surface of a solid adsorbate. In the first of the quoted papers, full equivalence between the thermodynamic-statistical method and the description based on the laws of chemical equilibrium was proved, with the considerations limited to the case of monolayer adsorption. The aim of the second article was, however, to demonstrate the fundamental possibility of extending the description to the case of a multilayer adsorption phase without the need to assume, as Berezin and Kisielev did [3], different models of the phenomenon for, respectively, the first and the subsequent adsorption layers. The presented solution was obtained assuming "horizontal" adsorbate-adsorbate dimerisation in the whole, infinitely multi-layered adsorption phase. The equilibrium state was described using, without modification, the Berezin and Kisielev schemes [3-5]. In the conclusion of the paper, besides the evaluation of the above-mentioned assumptions, it was emphasised that the finite form of the solution required the adoption of the physically unobvious condition of convergence of the respective infinite series.

This paper, continuing the subject of multilayer adsorption, presents at the same time a proposal of freeing the model from two of the above three limitations. Considerations (still) concerning the dimerisation of adsorbed molecules are based on a modified quasichemical scheme, in which the number of layers can take any value (finite or infinite). Moreover, in the final adsorption equation, the aspect of branched topography of adsorption sites, absent from Berezin's and Kisielev's concepts, was also taken into account.

The image of the adsorption phase adopted in the paper is based on the following assumptions:

- the surface area of the solid adsorbent contains $B$ identical binding sites as the centres for the location of the first adsorption layer,

- each adsorption site may bind at most one adsorbate molecule,

- the adsorption of a single gaseous component is, in principle, multi-layered, with only the lower immediate layer molecules being bound to the molecules in either of the further layers,

- $\quad$ similarly as in the Brunauer, Emmet and Teller model [6], the constant of equilibrium of adsorptive-adsorbate interactions is, regardless of the layer number, equal to the inverse pressure of the saturated steam of the adsorptive, $1 / p_{s}$,

- the molecules of each adsorption layer may be horizontally dimerised, with the process equilibrium constant, $\mathrm{K} 2$, being of equal value throughout the adsorption phase,

- the adsorptive is an ideal gas.

The types of complexes constituting the adsorption phase corresponding to the above image are presented in Figure 1. Each of the four illustration levels is supplemented with the symbols of these complexes (a) and the general code of the number of their molecules (b).

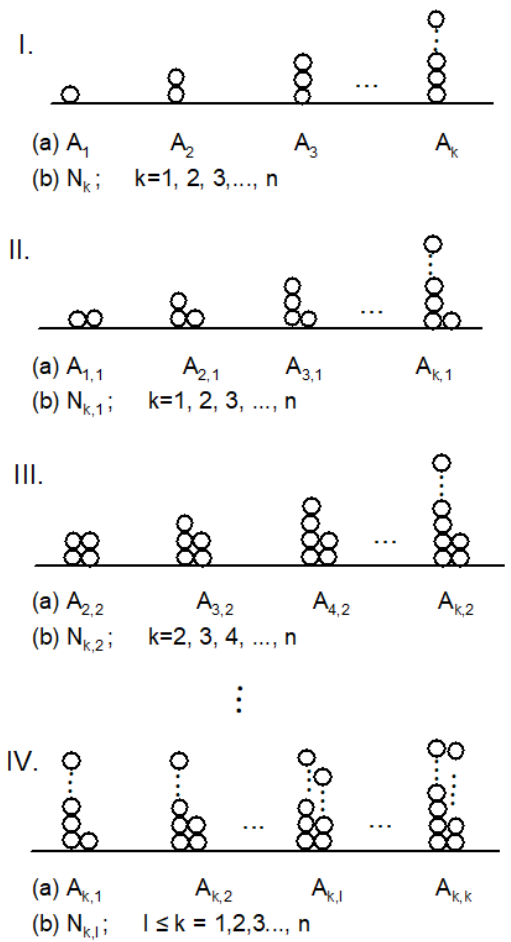

Fig. 1 Types of adsorption complexes and corresponding denotations. 
In the state of thermodynamic equilibrium of the system, all flows of extensive quantities disappear, and intensive equivalents of these quantities reach equilibrium values unchangeable over time. This does not exclude, of course, microscopic fluctuations of these values; however, averaged over time they are, according to the theory [7], inversely proportional to the number of molecules, and thus irrelevant in macroscopic systems. In our case, this means that both the adsorptive pressure and the concentrations of individual adsorption complexes (expressed, for example, in molar or molecular fractions) are determined by appropriate isothermal constants, i.e. the Langmuir constant, K1, the dimerisation constant K2 and the condensation constant $1 / \mathrm{ps}$. According to the presented concept, the basis for formulating expressions for these constants is the following diagram of intermolecular interactions in the adsorption system; (I) primary interactions (adsorptive-adsorbent):

$$
M+S \underset{\leftarrow}{\longrightarrow} A_{1}
$$

where $\mathrm{M}$ and $\mathrm{S}$ are respectively the adsorptive molecule and the free binding area on the surface of the adsorbent.

In the state of equilibrium [1]

$$
K_{1}=\frac{N_{1}}{p\left(B-N^{\prime}\right)}
$$

where $\mathrm{N}$ ' is the number of adsorption molecules in the first adsorption layer.

(II) secondary interactions (adsorptive-adsorbate and adsorbate-adsorbate):

$$
\begin{aligned}
& M+A_{1} \rightarrow A_{2} \\
& M+A_{2} \underset{\leftarrow}{\leftrightarrows} A_{3} \\
& \vdots \\
& M+A_{n-1 \leftarrow} A_{n} \\
& A_{1}+A_{1} \rightarrow \overleftarrow{A}_{1,1} \\
& M+A_{1,1} \underset{\leftarrow}{\rightarrow} A_{2,1} \\
& M+A_{2,1} \rightarrow A_{3,1} \\
& \text { ! } \\
& M+A_{n-1,1} \rightarrow A_{n, 1} \\
& M+A_{2,1} \rightarrow A_{2,2} \\
& M+A_{3,1} \leftrightarrows A_{3,2} \\
& M+A_{3,2} \stackrel{\leftrightarrows}{\leftrightarrows} A_{3,3} \\
& \text { : } \\
& M+A_{n, 1} \rightarrow A_{n, 2} \\
& M+A_{n, 2} \rightarrow A_{n, 3} \\
& M+A_{n, n-1} \underset{\leftarrow}{\leftarrow} A_{n, n}
\end{aligned}
$$

In accordance with assumption (iv) and (v), the expressions of equilibrium constants are, in the order of the above stoichiometric equations, as follows:

$$
\begin{aligned}
& \frac{1}{p_{s}}=\frac{N_{2}}{p N_{2}} \\
& \frac{1}{p_{s}}=\frac{N_{3}}{p N_{2}} \\
& \vdots \\
& \frac{1}{p_{s}}=\frac{N_{n}}{p N_{n-1}}
\end{aligned}
$$

$$
\begin{aligned}
& K_{2}=\frac{N_{1,1}}{N_{1}^{2}}\left(B-N^{\prime}+N^{a s}\right) \\
& \frac{1}{p_{s}}=\frac{N_{2,1}}{p N_{1,1}} \\
& \frac{1}{p_{s}}=\frac{N_{3,1}}{p N_{2,1}} \\
& \vdots \\
& \frac{1}{p_{s}}=\frac{N_{n, 1}}{p N_{n-1,1}} \\
& \frac{1}{p_{s}} K_{2}=\frac{N_{2,2}}{p N_{2,1}} \\
& \frac{1}{p_{s}} K_{2}=\frac{N_{3,2}}{p N_{3,1}} \\
& \frac{1}{p_{s}} K_{2}=\frac{N_{3,3}}{p N_{3,2}} \\
& \vdots \\
& \frac{1}{p_{s}} K_{2}=\frac{N_{n, 2}}{p N_{n, 1}} \\
& \frac{1}{p_{s}} K_{2}=\frac{N_{n, 3}}{p N_{n, 2}} \\
& \vdots \\
& \frac{1}{p_{s}} K_{2}=\frac{N_{n, n}}{p N_{n, n-1}}
\end{aligned}
$$

It is important to underline here that equation (5) expresses the second, next to the assumption (v), difference between our description and the concept of Berezin and Kisielev. The parenthesis to the right of this equation contains the number of molecules of all constituents of the adsorption equilibrium, i.e. the free binding sites in the number $\mathrm{B}-\mathrm{N}^{\prime}$ and the individual complexes in the number $\mathrm{N}$ as. Detailed justification for considering this factor is presented in our earlier publication [1]. We only note here that without it the constant K2 would not be an intensive quantity.

According to the assumed image of the adsorption phase (Fig. 1), the total number of adsorbate molecules, $\mathrm{N}$, is represented by the expression:

$N=\sum_{k=1}^{n} k N_{k}+\sum_{l \leq k=1}^{n}(k+l) N_{k, l}$

$\mathrm{N}^{\prime}$ and $\mathrm{Nas}$, on the other hand, are given in the formulas:

$N^{\prime}=\sum_{k=1}^{n} N_{k}+2 \sum_{l \leq k=1}^{n} N_{k, l}$

and

$N^{a s}=\sum_{k=1}^{n} N_{k}+\sum_{l \leq k=1}^{n} N_{k, l}$

The specification of the above relationships is possible thanks to the set of simultaneous equations (2)(14). Aside from tedious transformations, we can write down:

$N=N_{1} Z+N_{1,1} Y$,

$N^{\prime}=N_{1} W+N_{1,1} X$

and

$N^{a s}=N_{1} W+\frac{1}{2} N_{1,1} X$

where for $\mathrm{n}$ adsorption layers

$$
\begin{aligned}
& W=\frac{1-h^{n}}{1-h} \\
& X=\frac{2}{1-h},\left[\frac{1-\left(K_{2} h^{2}\right)^{n}}{1-K_{2} h^{2}}-h^{n} \frac{1-\left(K_{2} h\right)^{n}}{1-K_{2} h}\right]
\end{aligned}
$$


$Y=\frac{1}{(1-h)^{2}}\left\{2(1-h) K_{2} h^{2} \frac{1-n\left(K_{2} h^{2}\right)^{n-1}+(n-1)\left(K_{2} h^{2}\right)^{n}}{\left(1-K_{2} h^{2}\right)^{2}}+(2-\right.$

h) $\frac{1-\left(K_{2} h^{2}\right)^{n}}{1-K_{2} h^{2}}-h^{n}\left[(1 h) K h \frac{1-n\left(K_{2} h\right)^{n-1}+(n-1)\left(K_{2} h\right)^{n}}{\left(1-K_{2} h\right)^{2}}(2+\right.$

$\left.\left.n+(n+1) h) \frac{1-\left(K_{2} h\right)^{n}}{1-K_{2} h}\right]\right\}$

and

$Z=\frac{1-(n+1) h^{n}+n h^{n+1}}{(1-h)^{2}}$
The $\mathrm{h}$ above is the relative pressure of the adsorptive (as defined, $\mathrm{h}=\mathrm{p} / \mathrm{p}_{\mathrm{s}}$ ). Formulas (21)-(24) take a less complex form for the first few $n$ values, and also for $\mathrm{n} \rightarrow \infty$. In the latter case, however, the condition $\left(\mathrm{K}_{2} \mathrm{~h}^{2}\right)<1$ applies, which, in view of the double inequality, $0<\mathrm{h} \leq 1$, means, for the full $\mathrm{h}$ range, an even greater restrictiveness, namely: $\mathrm{K}_{2}<1$

The expressions corresponding to several illustrative $\mathrm{n}$ values are shown in Table 1.

Examples of the forms of functions (21)-(24).

\begin{tabular}{|c|c|c|c|c|}
\hline & \multicolumn{4}{|l|}{ Function } \\
\hline & $\mathrm{W}$ & $\mathrm{X}$ & $\mathrm{Y}$ & $\mathrm{Z}$ \\
\hline \multicolumn{5}{|l|}{$\mathrm{n}$} \\
\hline 1 & 1 & 2 & 2 & 1 \\
\hline 2 & $1+\mathrm{h}$ & $2\left(1+h+K_{2} h^{2}\right)$ & $2+3 h+4 K_{2} h^{2}$ & $1+2 \mathrm{~h}$ \\
\hline 3 & $1+\mathrm{h}+\mathrm{h}^{2}$ & $2\left(1+h+h^{2}+K_{2} h^{3}+K_{2}^{2} h^{4}\right)$ & $2+3 h+4 h^{2}+5 K_{2} h^{3}+6 K_{2}^{2} h^{4}$ & $1+2 h+3 h^{2}$ \\
\hline \multirow[t]{2}{*}{$\infty$} & 1 & 2 & $2-h\left(1+K_{2} h^{2}\right)$ & 1 \\
\hline & $\overline{1-h}$ & $\overline{(1-h)\left(1-K_{2} h^{2}\right)}$ & $\overline{\left[(1-h)\left(1-K_{2} h^{2}\right)\right]^{2}}$ & $\overline{(1-h)^{2}}$ \\
\hline
\end{tabular}

The next stage of model formulation requires the expression $\mathrm{N}_{1,1}$ through $\mathrm{N}_{1}$. For this purpose, using equations (19) and (20), we write equation (5) as follows:

$K_{2}=\frac{N_{1,1}}{N_{1}^{2}}\left(B-\frac{1}{2} N_{1,1} X\right)$

The solution to this equation for $\mathrm{N}_{1,1}$ is as follows:

$N_{1,1}=\frac{B-\sqrt{B^{2}-2 K_{2} X N_{1}^{2}}}{X}$

A simple substitution of the result (25) to formula (18) would produce a specific form of expression on $\mathrm{N} 1$ as a function of the total number of adsorbate molecules, N. Namely, it would be a solution corresponding to the model of only linear adsorbate-adsorbate association, i.e. consistent with the concept of Berezin and Kisielev [3]. Extending the description to a case of branched topography of binding sites requires, however, taking into account the fact that the adsorptive molecule being the $\mathrm{A}_{1}$ complex in one direction does not have to be it in the other directions (it may be a part of a more complex association in other directions). This issue was discussed in our previous publication [1], while formulating an appropriate generalisation. Taking this result into account leads to the following expression:

$N_{1}=\frac{N}{Z}\left[\frac{2-\frac{X}{Y} \Theta}{\sqrt{2 K_{2} \frac{Y}{Z^{2}}\left(2-\frac{X}{Y} \Theta\right)+1}+1-\frac{X}{Y} \Theta}\right]^{c / 2}$

where $\Theta=N / B$ is a dimensionless degree of filling the adsorbent surface, while $\mathrm{c}$ is the number of closest neighbours of the binding site (for linear topography c=2). Formulating the final adsorption equation requires only the determination of the relationship between $\mathrm{N}^{\prime}$ and $\mathrm{N}$. The form of this function results from the set of simultaneous equations (17) and (18) after the elimination of $\mathrm{N}_{1,1}$. After simple transformations,

$N^{\prime}=\frac{X}{Y} N\left[1-\frac{N_{1}}{N}\left(Z-\frac{W Y}{X}\right)\right]$ where $\mathrm{N}_{1}$ is given by equation (26).

In the end, the substitution of expressions (26) and (27) to equation (1) and the replacement of the adsorption pressure, $p$, with relative pressure, $h$, gives the adsorption equation in the following form:

where the isothermal constant $\mathrm{C}(\mathrm{T})$ is identical to the corresponding parameter in equation BET, whereas

$R=\left[\frac{2-\frac{X}{Y} \Theta}{\sqrt{2 \frac{K_{2} Y}{Z^{2}} \Theta\left(2-\frac{X}{Y} \Theta\right)+1}+1-\frac{X}{Y} \Theta}\right]^{c}$

Equation (28) together with substitutions (21)(24) and (29) is the final result of the considerations set out in this paper. The analysis of its boundary properties leads to the following conclusions:

a) For a monolayer $(n=1)$, equation (28) takes the form:

$p=\frac{\Theta}{K_{1}(1-\Theta)}\left[\frac{2-\Theta}{\sqrt{4 K_{2} \Theta(2-\Theta)+1}+1-\Theta}\right]^{c / 2}$

It is the formula that we presented in our publication concerning the equivalence of statistical and phenomenological methods in the description of monolayer adsorption [1], and which for $c=2$ and $\Theta<1$ transforms into an equation published more than 40 years ago by Karpiński and one of the authors of this article [8].

b) The absence of adsorbate adsorbate dimerisation $(\mathrm{K} 2=0)$ results in the following boundary forms of equation (28):

$$
\begin{gathered}
p=\frac{\Theta^{-}}{K_{1}(1-\Theta)} \quad \text { for } \mathrm{n}=1, \\
-\quad \text { for } 1<\mathrm{n}<\infty, \\
h \frac{1-h^{n}}{1-h}=\frac{\Theta^{\prime}}{C(T)\left(1-\Theta^{\prime}\right)}
\end{gathered}
$$


where

$$
\begin{aligned}
& \Theta^{\prime}=\Theta \frac{(1-h)\left(1-h^{n}\right)}{1-(n+1) h^{n}+n h^{n+1}} \\
& -\quad \text { for } \mathrm{n} \rightarrow \infty, \\
& h=\frac{\Theta(1-h)^{2}}{C(T)[1-\Theta(1-h)]}
\end{aligned}
$$

In these dependencies we can easily recognise the Langmuinr [9], BET (n) and BET ( $\infty$ ) [6] equations, respectively. Note that the assumption common to these equations: $K_{2}=0$ implies their independence from the topography of the binding sites of the adsorbent.
Based on the above analysis, it can be concluded that the proposed model, together with its solution (28), is the most general form of description of adsorbateadsorbate dimerisation in a multilayer adsorption phase localised on a homogeneous solid surface.

The result obtained opens the prospect of further theoretical research. From the point of view of the authors, it is particularly cognitively attractive to see higher factor combinations of adsorbate-adsorbate associations as well as partial mobility of the adsorption phase.

\section{REFERENCES}

1. Garbacz JK., Kozakiewicz M. The equivalence of quasi-chemical and statistical description of adsorbate molecule association in a localised adsorption monolayer on a homogeneous solid surface. PolHypRes 2018 Vol. 65 Issue 4 pp. 25 - 38 DOI: 10.2478/phr-2018-0021;

2. Garbacz JK., Karpiński K., Kozakiewicz M. Dimerization of molecules in a multilayer adsorption phase on homogeneous surfaces of solid adsorbent. PolHypRes 2019 in press;

3. Berezin G.I., Kiselev A.V., Adsorbate-adsorbate association on a homogenous surface of a nonspecific adsorbent. Journal of Colloid and Interface Science, 1972. 38(1): p. 227;

4. Berezin G.I., Kiselev A.V. A model of an associated two-dimensional gas on a homogeneous surface Journal of Colloid and Interface Science Volume 46, Issue 2, February 1974, Pages 203-211;

5. Garbacz J.K., About adsorbate-adsorbate association on homogeneous adsorbent surfaces. Journal of Colloid and Interface Science, 1975 . 51(2): p. 352-353;

6. Brunauer S., Emmett PH., Teller E. Adsorption of gases in multimolecular layers. J Am Chem Soc. 1938;60(2):309-19.

7. Huang, K., Statistical mechanics. 1963, New York: Wiley. 470;

8. Karpinski, K., Garbacz JK., General formulation of description of adsorption in a localized monolayer on homogeneous surfaces. Roczniki Chemii, 1974. 48(5): p. 803-809;

9. Langmuir I. The adsorption of gases on plane surfaces of glass, mica and platinum. J Am Chem Soc. 1918;40(9):1361-403.

prof. dr hab. Jerzy K. Garbacz

Uniwersytet Technologiczno-Przyrodniczy w Bydgoszczy,

Wydział Budownictwa, Architektury i Inżynierii Srodowiska,

Katedra Ekoinżynierii i Fizykochemii Środowiska

ul. Sucha 9, 85-789 Bydgoszcz

(52) $3408440 / 3408445$

jerzy.garbacz@utp.edu.pl 